\title{
ITERATING MAPS ON CELLULAR COMPLEXES
}

\author{
STEPHEN J. WILLSON
}

\begin{abstract}
Let $K$ be a finite simplicial complex and $f: K \rightarrow K$ be a "skeletal" map. A digraph $D$ is defined whose vertices correspond to the simplexes of $K$ and whose arcs give information about the behavior of $f$ on the simplexes. For every walk in $D$ there exists a point of $K$ whose iterates under $f$ mimic the walk. Periodic walks are mimicked by a periodic point. Digraphs with uncountably many infinite walks are characterized; the corresponding maps $f$ exhibit complicated behavior.
\end{abstract}

\section{INTRODUCTION}

This paper concerns the iteration of maps defined on a suitable generalization of a simplicial complex. Suppose that $K$ is a finite simplicial complex (polyhedron) and $f: K \rightarrow K$ is a "skeletal" map (for example, a simplicial map). We are concerned with studying properties of the iterates $f, f^{2}, f^{3}, \ldots$ of $f$. Our basic tool will be a finite digraph $D$ which we call the "iteration digraph." The vertices of $D$ correspond to the simplexes of $K$, and the arcs of $D$ give information about the behavior of $f$ on the simplexes.

The theorems in this paper tend to reduce analytic or topological questions about $f$ to combinatorial questions about the digraph $D$. For example, given any infinite walk in $D$ there exists a point $x$ of $f$ whose successive iterates follow that walk in the sense that $f^{i}(x)$ is in the $i$ th simplex of the walk. Thus if $D$ exhibits a rich diversity of walks, $f$ in turn exhibits a rich diversity in the nature of the orbits of points.

In a broad sense, this paper is a contribution to the study of possibly chaotic dynamics (see [D]) in dimensions that may be higher than one. Chaos has been most thoroughly studied for maps on an interval. The material here is related to the "itineraries" of Milnor and Thurston [MT], also described in [CE] or [Gu]. It is also related to symbolic dynamics, as in [P], and Markov partitions, as in [B].

If $K$ is a triangulation of the closed unit interval, then the restriction that $f$ be "skeletal" is a strong constraint. It is nevertheless much more general than assuming that $f$ be a simplicial map. Moreover, our methods work in any dimension. Even in dimension one we obtain a very convenient tool in the iteration digraph $D$.

Received by the editors July 25, 1989 and, in revised form, April 2, 1990.

1980 Mathematics Subject Classification (1985 Revision). Primary 58F13; Secondary 05C20.

Key words and phrases. Simplicial complex, digraph, chaotic dynamics. 
The iteration digraph $D$ is defined as follows: A vertex $v$ of the digraph $D$ corresponds to a simplex $I(v)$ of $K$; there is an arc from $v$ to $w$ if and only if $f(I(v))$ contains $I(w)$. Suppose that in $D$ the infinite walk $\sigma=v_{0} v_{1} \ldots$ is given, where $v_{0}, v_{1}, \ldots$ is a succession of vertices. Let $C \sigma=\{x \in K$ : $f^{i}(x) \in I\left(v_{i}\right)$ for each $\left.i\right\}$. For skeletal maps we prove that $C \sigma$ will always be compact and nonempty. Conversely, if $x \in K$ then there exists an infinite walk $\sigma$ such that $x \in C \sigma$. Points are common to $C \sigma$ and $C \tau$ if and only if the walks $\sigma$ and $\tau$ have a common "restriction" in $D$. Hence information about the possible orbit types of points in $K$ may be obtained by studying the collection of walks in $D$.

If $\sigma$ is a finite walk and all vertices correspond to simplexes of dimension $k$, then $C \sigma$ is homeomorphic to a $k$-dimensional disk. This permits us to use Brouwer's Fixed Point Theorem to prove that if the infinite walk $\sigma$ is periodic, then $C \sigma$ contains a periodic point. Further information is needed to obtain that the walk and the periodic point have the same least period.

We say $f$ has rich orbit structure if $D$ has uncountably many distinct infinite walks. There is then much diversity in the behavior of points under iteration by $f$. Some equivalent conditions are given in $\S 4$, some sufficient conditions in $\S 5$, and some consequences (with mild additional assumptions) in $\S 6$. The consequences include a kind of sensitivity to initial conditions and the existence of periodic points with arbitrarily large least period. These are slightly modified concepts from those involved in "chaotic" behavior. In $\S 7$ we briefly describe a generalization of the results, and in $\S 8$ we present an example.

\section{SKeletal MAPS AND THE ITERATION DIGRAPH}

The basic objects we study are cellular complexes and skeletal maps. These are generalizations of simplicial complexes and simplicial maps.

Definition. A (closed) $m$-cell $I$ is a metric space homeomorphic to a standard closed $m$-dimensional simplex $\Delta^{m}$, the convex hull of $m+1$ independent points $v_{0}, \ldots, v_{m}$ in Euclidean space. The interior of $I$, denoted int $(I)$, consists of points in $I$ corresponding to points in $\Delta^{m}$ of form $\sum a_{i} v_{i}$ where $a_{i}>0$ and $\sum a_{i}=1$. The boundary of $I$, denoted $\mathrm{bd}(I)$, is the complement in $I$ of $\operatorname{int}(I)$. The dimension of the cell is $m$.

Definition. A cellular complex $K$ is a compact metric space for which there is a nested sequence of compact subspaces $K^{0} \subset K^{1} \subset \cdots \subset K^{m}=K$ for some $m$, where $K^{i}$ is called the $i$-skeleton of $K$, satisfying the following:

(1) $K^{0}$ consists of finitely many points, called 0 -simplexes.

(2) For each $i>0, K^{i}$ consists of $K^{i-1}$ together with finitely many $i$ simplexes $I_{1}, I_{2}, \ldots, I_{r}$ for some $r$ (depending on $i$ ).

(3) Each of the $i$-simplexes $I_{j}$ is the image of a one-to-one continuous map $\phi_{j}: \Delta^{i} \rightarrow K$ such that the boundary of $\Delta^{i}$ gets mapped into $K^{i-1}$. Thus each $i$-simplex is an $i$-cell.

(4) The interiors of the various $i$-simplexes are assumed to be pairwise disjoint and disjoint from $K^{i-1}$.

(5) For each $i>0$, the boundary of each $i$-simplex is precisely a union of simplexes of lower dimension. Each simplex in the boundary of the $i$-simplex is called a face of the $i$-simplex; and by convention each $i$-simplex is also a face of itself. Note that every point $x$ is in the interior of a unique simplex. 
Example. Any finite simplicial complex $K$ is a cellular complex using the standard notion of simplex.

Definition. Let $K$ be a cellular complex. A skeletal map $f: K \rightarrow K$ is a continuous function $f: K \rightarrow K$ such that

(1) for each $i, f\left(K^{i}\right) \subseteq K^{i}$;

(2) for each simplex $I$ of $K, f(I)$ is a union of simplexes of $K$;

(3) for each $k$ and for each $k$-dimensional simplex $I$ of $K$, either $f \mid I$ is one-to-one or else $f(I) \subseteq K^{k-1}$.

It follows from (2) that if $I$ and $J$ are simplexes of $K$ and $f(I)$ contains an interior point of $J$, then $f(I)$ contains all of $J$.

Example. Suppose that $L$ is a finite simplicial complex, $K=L^{\prime}$ is a subdivision of $L$, and $f: K \rightarrow L$ is a simplicial map. Then $f$ is a skeletal map on $K$. By the Simplicial Approximation Theorem [S] any continuous map $g: L \rightarrow L$ is homotopic to a simplicial map $f: L^{\prime} \rightarrow L$ for some subdivision of $L$; hence there are numerous skeletal maps. Note, however, that a skeletal map is more general than a simplicial map; for example, a skeletal map need not be linear on simplexes.

Standing assumption. Unless otherwise stated, all complexes in this paper are cellular and all maps are skeletal.

Definition. If $f$ is a skeletal map on the cellular complex $K$, we obtain the iteration digraph denoted $D$ as follows: There is one vertex for each simplex of $K$. Thus we may take the vertex set $V$ to be the index set for the set of simplices of $K$, and we shall write $I(v)$ for the simplex of $K$ corresponding to the vertex $v \in V$. There will be an arc from vertex $v$ to vertex $w$ if and only if $f(I(v))$ contains $I(w)$. We write the arc from $v$ to $w$ as $(v, w)$; if there is an arc from $v$ to $w$ we often write $v \rightarrow w$. It is possible that $D$ contains a directed loop $(v, v)$ at any $v$, but for any $v$ and $w$ there can be at most one $\operatorname{arc}(v, w)$ and at most one $\operatorname{arc}(w, v)$.

For an integer $k$, the $k$-iteration-digraph $D^{k}$ is the subdigraph of $D$ generated by all $k$-dimensional simplexes of $K$. Thus $v \in V$ is a vertex of $D^{k}$ if and only if $I(v)$ is a $k$-dimensional simplex of $K$; and $v \rightarrow w$ in $D^{k}$ if and only if both $v$ and $w$ are vertices of $D^{k}$ and $f(I(v))$ contains $I(w)$.

A finite walk in the digraph $D$ is a finite sequence $v_{0}, v_{1}, v_{2}, \ldots, v_{m}$ where each $v_{j}$ is a vertex of $D$ and for each $j, 0 \leq j \leq(m-1),\left(v_{j}, v_{j+1}\right)$ is an arc of $D$. The length of the walk is $m$, the number of arcs. An infinite walk is a similar infinite sequence of vertices. The set of all infinite walks in $D$ will be denoted $\Sigma^{D}$. A typical element of $\Sigma^{D}$ will be denoted $\sigma=v_{0} v_{1} \ldots$.

If $\sigma=v_{0} v_{1} \cdots$ is a finite or infinite sequence of vertices of $D$, we let $C \sigma=\left\{x \in K: f^{i}(x) \in I\left(v_{i}\right)\right.$ for each $\left.i\right\}$. Here $f^{i}(x)$ denotes the result of iterating the function $i$ times, so for example $f^{3}(x)=f(f(f(x)))$. By convention $f^{0}(x)=x$ for all $x$.

Proposition 2.1. Suppose $\sigma=v_{0} v_{1} \cdots v_{m}$ is a walk in $D^{k}$ of length $m$. Then for each $i$ such that $0 \leq i \leq m$ the function $f^{i}$ gives a homeomorphism of $C \sigma=C v_{0} v_{1} \cdots v_{m}$ onto $C v_{i} \cdots v_{m}$.

Proof. We use induction on $m$, the case $m=0$ being immediate. Assume the result for $m-1$. Since $v_{0} \rightarrow v_{1}$ in $D^{k}$, we know that $I\left(v_{0}\right)$ and $I\left(v_{1}\right)$ 
are $k$-simplexes and $f\left(I\left(v_{0}\right)\right)$ contains $I\left(v_{1}\right)$. Since $f$ is skeletal and $f\left(I\left(v_{0}\right)\right)$ meets the interior of the $k$-simplex $I\left(v_{1}\right)$, it follows that $f \mid I\left(v_{0}\right)$ is one-to-one hence a homeomorphism onto its image. Clearly

$$
C v_{0} v_{1} \cdots v_{m}=\left(f \mid I\left(v_{0}\right)\right)^{-1}\left(C v_{1} \cdots v_{m}\right)
$$

so $f$ gives a homeomorphism of $C v_{0} v_{1} \cdots v_{m}$ onto $C v_{1} \cdots v_{m}$. By the induction hypothesis, for each $i$ such that $1 \leq i \leq m$ the function $f^{i-1}$ gives a homeomorphism of $C v_{1} v_{2} \cdots v_{m}$ onto $C v_{i} v_{i+1} \cdots v_{m}$. Composing these maps, we obtain the result.

Corollary 2.2. If $\sigma$ is a walk in $D^{k}$ of length $m$, then $C \sigma$ is a k-cell. Proof. The map $f^{m}$ gives a homeomorphism of $C \sigma$ onto the $k$-cell $I\left(v_{m}\right)$.

Corollary 2.3. If $\sigma=v_{0} v_{1} \cdots v_{m}$ is a finite walk in $D^{k}$, then there exists $x \in K$ such that for each $i$ satisfying $0 \leq i \leq m$ we have $f^{i}(x) \in \operatorname{int}\left(I\left(v_{i}\right)\right)$.

Proof. By Invariance of Domain, each point $x$ in int $(C \sigma)$ maps by the homeomorphism $f^{i}$ into the interior of $C v_{i} \cdots v_{m}$ and hence into int $\left(I\left(v_{i}\right)\right)$.

Corollary 2.4. If $\sigma$ is an infinite walk in $D^{k}$, then $C \sigma$ is compact, connected, and nonempty.

Proof. If $\sigma=v_{0} v_{1} \cdots$, then for $m \geq 0$ define the finite walks $\sigma_{m}=v_{0} v_{1} \cdots v_{m}$. Clearly $C \sigma$ is the intersection of the nested sets $C \sigma_{0} \supset C \sigma_{1} \supset C \sigma_{2} \supset \cdots$. By (2.2) each of these is a $k$-cell, hence compact, connected, and nonempty. The common intersection is then nonempty by the finite intersection property of compact sets, and the other properties follow similarly.

Theorem 2.5. If $\sigma \in \Sigma^{D}$ is an infinite walk in $D$, then $C \sigma$ is compact and nonempty.

Proof. Using the same notation as above, $C \sigma$ is the intersection of the nested sets $C \sigma_{0} \supset C \sigma_{1} \supset \cdots$. Since $v_{0} \rightarrow v_{1}$ we know $f\left(I\left(v_{0}\right)\right)$ contains $I\left(v_{1}\right)$ so $C \sigma_{1}=C v_{0} v_{1}$ is compact and nonempty. Similarly $C v_{1} v_{2}$ is compact and nonempty because $v_{1} \rightarrow v_{2}$; and since $f\left(I\left(v_{0}\right)\right)$ contains $I\left(v_{1}\right)$ it contains the compact subset $C v_{1} v_{2}$. Hence $C \sigma_{2}=C v_{0} v_{1} v_{2}$ is compact and nonempty. Repeating the argument we obtain that each set $C \sigma_{m}$ is compact and nonempty, whence so is their nested intersection $C \sigma$.

Definition. If $x \in K$, we write $J(x)=I$ where $I$ is the unique simplex such that $x \in \operatorname{int}(I)$. The standard walk of $x$, denoted $\phi(x)$, is then defined by $\phi(x)=v_{0} v_{1} \cdots \in \Sigma^{D}$ where $I\left(v_{i}\right)=J\left(f^{i}(x)\right)$ for $i \geq 0$. Since $f\left(f^{i}(x)\right)$ is in $\operatorname{int}\left(I\left(v_{i+1}\right)\right)$ it follows that $f\left(I\left(v_{i}\right)\right)$ contains $I\left(v_{i+1}\right)$, so $v_{i} \rightarrow v_{i+1}$ and $\sigma$ is indeed a walk in $D$.

The standard walk yields a correspondence (not necessarily one-to-one) between points of $K$ and walks in $D$, which we summarize as follows:

Proposition 2.6. If $x \in K$, then there is a walk $\sigma \in \Sigma^{D}$ such that $x \in C \sigma$. Conversely, if $\sigma \in \Sigma^{D}$ is a walk in $D$, then there exists $x \in K$ such that $x \in C \sigma$.

It is not true that for every infinite walk $\sigma$ there is an $x \in K$ such that $\sigma=\phi(x)$. For example, suppose that $K$ is the interval $[0,1]$ with 0 simplexes 
0 and 1 and with 1 -simplex $a=[0,1]$. Let $f$ be the identity map on $K$. Then the infinite walk $\sigma=a 111111 \cdots$ in $D$ is such that only 1 lies in $C \sigma$ and yet clearly $\phi(1)=11111 \cdots$.

Recall that if $I$ is a $k$-simplex of $K$, then $I$ is a $k$-cell and its boundary $\mathrm{bd}(I)$ is a union of simplexes of dimension $k-1$ or lower. Each simplex contained in $I$ is called a face of $I$, and by convention $I$ is also a face of itself.

Definition. Let $\sigma=v_{0} v_{1} \cdots v_{m}$ [resp. $v_{0} v_{1} \cdots$ ] be a finite [resp. infinite] walk in $D$. Then another walk $\tau=w_{0} w_{1} \cdots w_{m}$ [resp. $\left.w_{0} w_{1} \cdots\right]$ in $D$ is a restriction of $\sigma$ provided that for all $i, I\left(w_{i}\right)$ is a face of $I\left(v_{i}\right)$. It is immediate that $C \tau$ is then a subset of $C \sigma$.

Lemma 2.7. Suppose $x \in C \sigma$ where $\sigma$ is an infinite walk in $D$. Then the standard walk $\phi(x)$ is a restriction of $\sigma$.

Proof. Let $\sigma=v_{0} v_{1} \cdots$ and let $\phi(x)=w_{0} w_{1} \cdots$. Note that $f^{i}(x) \in \operatorname{int}\left(I\left(w_{i}\right)\right)$ while $f^{i}(x) \in I\left(v_{i}\right)$. Since $I\left(v_{i}\right)$ contains an interior point of $I\left(w_{i}\right)$, it must contain all of $I\left(w_{i}\right)$. Hence $I\left(w_{i}\right)$ is a face of $I\left(v_{i}\right)$.

Proposition 2.8. Let $\sigma$ and $\tau$ be infinite walks in $D$. Then $C \sigma$ and $C \tau$ have a point in common if and only if $\sigma$ and $\tau$ have a common restriction.

Proof. Suppose $x$ lies in the intersection of $C \sigma$ and $C \tau$. Then $\phi(x)$ is a common restriction of $\sigma$ and $\tau$. Conversely, suppose that $\mu$ is a common restriction of $\sigma$ and $\tau$. Choose $x \in C \mu$ by (2.5). Then $x$ clearly lies in $C \sigma$ and $C \tau$.

Corollary 2.9. Suppose that $\sigma=v_{0} v_{1} \cdots$ and $\tau=w_{0} w_{1} \cdots$ are walks in $D$ and that for some $i, I\left(v_{i}\right)$ and $I\left(w_{i}\right)$ are disjoint. Then $C \sigma \cap C \tau=\varnothing$.

The significance of these results is that they reduce topological questions (about the intersections of compact subsets) to combinatorial questions (about the existence of common restrictions to walks).

\section{PERIODIC POINTS}

A point $x \in K$ is periodic of period $p>0$ if for all $i, f^{i+p}(x)=f^{i}(x)$. The smallest such $p$ will be called the least period of $x$. Similarly an infinite walk $\sigma=v_{0} v_{1} \ldots$ is periodic of period $p$ if for each $i, v_{i}=v_{i+p}$, and the smallest such $p$ is the least period of $\sigma$.

Clearly the standard walk $\phi(x)$ of a point $x$ which is periodic of period $p$ is a periodic walk of period $p$. The least period of $x$, however, need not be the same as the least period of $\phi(x)$. For example, if $K$ is a standard 2-simplex with its simplicial faces and $f$ is the natural rotation by 120 degrees, then for all interior points $x$ except the center of rotation the least period of $x$ is 3 while the least period of $\phi(x)$ is 1 .

Theorem 3.1. Let $\sigma=v_{0} v_{1} \cdots$ be an infinite walk in $D^{k}$ which is periodic of least period $p$. Then there exists a point $x \in C \sigma$ which is periodic of period $p$ (but not necessarily least period $p$ ). If $x \in \operatorname{int}\left(I\left(v_{0}\right)\right)$, then $x$ has least period $p$. If $x \in \operatorname{bd}\left(I\left(v_{0}\right)\right)$ then the standard walk $\phi(x)$ is a restriction of $\sigma$ of period $p$ in $D^{j}$ for some $j$ satisfying $j<k$. 
Proof. Note $v_{0}=v_{p}$. By (2.1) the map $g=f^{p} \mid C v_{0} \cdots v_{p}$ defines a homeomorphism of $C v_{0} \cdots v_{p}$ onto $C v_{p}=C v_{0}$. The inverse map $g^{-1}$ is then a continuous map of the $k$-cell $C v_{0}$ into itself since $C v_{0} \cdots v_{p}$ is a subset of $C v_{0}$. By the Brouwer Fixed Point Theorem, $g^{-1}$ has a fixed point $x$, whence $f^{p}(x)=x$ and $x$ is a periodic point in $C v_{0} \cdots v_{p}$ of period $p$. It is then obvious that $x \in C \sigma$.

If $x \in \operatorname{int}\left(I\left(v_{0}\right)\right)$, then for all $i, f^{i}(x) \in \operatorname{int}\left(I\left(v_{i}\right)\right)$. (Otherwise for some $i<$ $p$ we would have $f^{i}(x) \in \operatorname{bd}\left(I\left(v_{i}\right)\right)$. Since $f^{p-i}$ yields a homeomorphism of $C v_{i} \cdots v_{p}$ onto $C v_{p}=C v_{0}$ and $f^{i}(x) \in \operatorname{bd}\left(C v_{i} \cdots v_{p}\right)$, invariance of domain would imply that $x=f^{p-i}\left(f^{i}(x)\right)$ is in $\operatorname{bd}\left(I\left(v_{0}\right)\right)$, a contradiction.) Hence $v_{i}$ is uniquely determined by the condition $f^{i}(x) \in \operatorname{int}\left(I\left(v_{i}\right)\right)$. If $x$ had period $q$ strictly smaller than $p$, then it follows that $\sigma$ would have period $q$ as well, a contradiction. Hence $x$ has least period $p$.

If $x \in \operatorname{bd}\left(I\left(v_{0}\right)\right)$ then $\phi(x)=w_{0} w_{1} \cdots$ is a periodic walk in $D$ which by (2.7) is a restriction of $\sigma$. Let $j$ be the dimension of $I\left(w_{0}\right)$. Then all $I\left(w_{i}\right)$ have dimension $j$ also, since the dimension of $I\left(w_{i}\right)$ can never strictly increase but $\phi(x)$ is periodic. Thus $\phi(x)$ is a walk in $D^{j}$.

Example. We give an example of a cellular complex and skeletal map such that the only periodic points are fixed (i.e., of period 1) while there exist periodic walks of arbitrary period.

We define $X=[0,2] \times[0,1]$ and $K=X / \sim$ using the quotient topology, where $\sim$ is the equivalence relation $(x, 0) \sim(0,0)$ and $(x, 1) \sim(0,1)$ for all $x \in[0,2]$. Choose a monotone increasing homeomorphism $k$ of $[0,1]$ onto itself such that $k(x)>x$ if $0<x<1$, but $k(0)=0$ and $k(1)=1$. Define the map $f: K \rightarrow K$ which is induced from the formula on $X$ given by $f(x, y)=(2 x, k(y))$ if $0 \leq x \leq 1$ while $f(x, y)=(4-2 x, k(y))$ if $1 \leq x \leq 2$.

Now $K$ has a cellular structure as follows. It has two 0 -simplexes $a$ and $b$, where $a$ is the equivalence class of $(0,0)$ and $b$ is the class of $(0,1)$. It has three 1 -simplexes $c, d$, $e$, each with $a$ and $b$ as the two ends: $c$ is the image of all points of form $(0, y), d$ is the image of all points of form $(1, y)$, and $e$ corresponds to all points of form $(2, y)$. And $K$ has two 2-simplexes $g$ and $h$, where $g$ corresponds to all points of form $(x, y)$ with $0 \leq x \leq 1$ and $h$ corresponds to all points of form $(x, y)$ with $1 \leq x \leq 2$.

It is easy to see that $f: K \rightarrow K$ is a skeletal map. But from the formula for $f$ it is also clear that the only periodic points correspond to $(x, y)$ where $k(y)=y$, whence $y=0$ or 1 . Hence the only periodic points are $a$ and $b$, which are fixed.

Note that $D^{2}$ has arcs $g \rightarrow g, g \rightarrow h, h \rightarrow g, h \rightarrow h$. Hence $D^{2}$ possesses periodic walks of arbitrary least period $p>1$, for example $\sigma=$ $g^{p-1} h g^{p-1} h \cdots$. The only periodic points in $C \sigma$ are $a$ and $b$, which have least period 1. Hence one cannot find a point in $C \sigma$ of least period $p$.

Corollary 3.2. Let $\sigma \in \Sigma^{k}$ be a periodic walk of least period $p$. Suppose that $\sigma$ has no proper periodic restrictions (i.e., there is no periodic restriction $\tau$ of $\sigma$ of dimension strictly less than $k)$. Then $C \sigma$ contains a point $x \in \operatorname{int}\left(I\left(v_{0}\right)\right)$ of least period $p$.

The significance of (3.2) is that the analytic question of whether a periodic 
point of a certain period exists is often thereby reduced to a combinatorial question. The following results are more specific consequences:

Corollary 3.3. Let $\sigma=v_{0} v_{1} \cdots$ be a periodic infinite walk in $D^{k}$ of least period $p$. Suppose that $p$ is prime and assume that there is no fixed point of $f$ simultaneously in all simplexes $I\left(v_{i}\right)$. Then there exists a point $x$ of least period $p$ in $C \sigma$.

Proof. Choose $x \in C \sigma$ with period $p$. If $x$ has least period strictly less than $p$, then since $p$ is prime, the least period must be 1 and $x$ must be a fixed point of $f$. But then $x$ is a fixed point simultaneously in all simplexes $I\left(v_{i}\right)$, contrary to assumption.

Corollary 3.4. Let $\sigma=v_{0} v_{1} \cdots$ be an infinite walk in $D^{k}$ of least period $p$ where $p$ is prime. Suppose that there is no face a which is common to $I\left(v_{0}\right), I\left(v_{1}\right), \ldots, I\left(v_{p-1}\right)$ and satisfies that $a \rightarrow a$. Then $C \sigma$ contains a point $x$ of least period $p$.

Proof. Let $x$ be a point of period $p$ in $C \sigma$. Since $p$ is prime, if $x$ does not have least period $p$, its least period must be 1 . Hence the standard walk of $x$ must have form $\phi(x)=a a a \cdots$ for some face $a$ which contradicts the hypothesis.

Corollary 3.5. Let $\sigma=v_{0} v_{1} \cdots$ be a periodic walk in $D^{k}$ of least period $p$. Suppose that there is an $i$ so $f\left(\operatorname{int}\left(I\left(v_{i}\right)\right)\right)$ contains $I\left(v_{i+1}\right)$. Then $C \sigma$ contains a point of least period $p$.

Proof. Let $x \in C \sigma$ have period $p$. Let the standard walk for $x$ be $\phi(x)=$ $w_{0} w_{1} \cdots$. Since $f \mid I\left(v_{i}\right)$ is a homeomorphism and $f^{i+1}(x)$ is in $I\left(v_{i+1}\right)$, it follows that $f^{i}(x)$ is in $\operatorname{int}\left(I\left(v_{i}\right)\right)$ so $w_{i}=v_{i}$. The dimension of $I\left(w_{j}\right)$ cannot increase with $j, x$ is periodic, and the dimension of $I\left(w_{i}\right)=I\left(v_{i}\right)$ is $k$, so it follows that the dimension of $I\left(w_{j}\right)$ is $k$ for all $j$. Hence $w_{0}=v_{0}$ and $x$ is in $\operatorname{int}\left(I\left(v_{0}\right)\right)$, whence the least period of $x$ is $p$ by $(3.1)$.

Remark. It does not follow from (3.1) that if $\sigma$ has period $p$ then all periodic points in $C \sigma$ have period $p$. Indeed it is possible for $\sigma$ to have period 1 and yet contain periodic points of arbitrarily large least period. This does not, however, occur in one-dimensional complexes:

Proposition 3.6. Suppose $\sigma=v_{0} v_{1} \cdots$ is a periodic walk in $D^{1}$ with least period $p$. If $x \in C \sigma$ is a periodic point, then $x$ has period $p$ or $2 p$.

Proof. Let $x \in C \sigma$ be periodic. The map $f^{p} \mid C v_{0} v_{1} \cdots v_{p} \rightarrow C v_{p}=C v_{0}$ is a homeomorphism by (2.1). Suppose first that this homeomorphism preserves orientation, i.e., it is a strictly increasing map. If $x<f^{p}(x)$ and $x \in C \sigma$, then it follows that $x, f^{p}(x), f^{2 p}(x), \ldots$ are all in $C \sigma$, so $x<f^{p}(x)<f^{2 p}(x)<$ $f^{3 p}(x)<\cdots$; whence $x$ could not be periodic. Similarly if $x>f^{p}(x)$ and $x \in C \sigma$ then $x$ cannot be periodic. Thus $x=f^{p}(x)$ and $x$ must have period $p$.

Suppose next that the homeomorphism reverses orientation. Then

$$
f^{2 p} \mid C v_{0} v_{1} \cdots v_{2 p} \rightarrow C v_{0}
$$

is a homeomorphism that preserves orientation. Hence the first case applies and proves that $x$ must have period $2 p$. 


\section{RICH ORBIT STRUCTURE}

Let $K$ be a cellular complex and $f: K \rightarrow K$ be a skeletal map. Walks in the iteration digraph correspond to the possibilities for the orbits of points in $K$. In this section we give conditions under which there is a rich diversity of such orbits. Maps with these conditions are related to "chaotic" maps.

Let $A$ denote the adjacency matrix for the digraph $D$. If $n$ is the number of vertices of $D$ (hence the number of simplexes of $K$ ), then $A$ is an $n$ by $n$ matrix. The entry $A_{i j}$ equals 1 if $(i, j)$ is an arc of $D$ and equals 0 otherwise. It is well known that the entry $\left(A^{m}\right)_{i j}$ equals the number of walks of length precisely $m$ which start at $i$ and end at $j$. Let $w(m)$ denote the total number of walks in $D$ of length $m$. Thus $w(m)=\sum\left(A^{m}\right)_{i j}$ where the summation is over all $i$ and all $j$.

A cycle in the digraph $D$ is a walk $v_{0} v_{1} \cdots v_{m}$ in $D$ such that $v_{0}=v_{m}$ but the vertices $v_{0}, v_{1}, \ldots, v_{m-1}$ are all distinct.

Since $A$ has all entries nonnegative, there exists a dominant eigenvalue $\lambda$ for $A$. It is known [Ga] that $\lambda \geq 0$ and every eigenvalue $\mu$ of $A$ satisfies that $|\mu| \leq \lambda$. Furthermore, $\lambda$ has an eigenvector $v \geq 0$. (To say $v \geq w$ where $v$ and $w$ are vectors or matrices is to say for each component $v_{i} \geq w_{i}$; similarly $v>w$ if for each component $v_{i}>w_{i}$.)

Suppose $a(m)$ is a sequence of nonnegative numbers and $p$ is constant. We write $a(m)=O\left(m^{p}\right)$ if there is a constant $C$ so $a(m) \leq C m^{p}$ for all positive integers $m$. We then say that $a(m)$ has polynomial growth with $m$. If there is a positive constant $C$ and a number $\lambda>1$ such that $a(m)>C \lambda^{m}$, then we say that $a(m)$ has exponential growth with $m$.

Theorem 4.1. The following conditions are equivalent:

(1) $D$ has uncountably many infinite walks.

(2) The number $w(m)$ of walks in $D$ of length $m$ has exponential growth with $m$.

(3) $A$ has dominant eigenvalue $\lambda>1$.

(4) Some vertex of $D$ is on two distinct cycles.

Definition. If the map $f$ satisfies that $D$ has any of these properties, we will say $f$ has rich orbit structure.

If $f$ does not have rich orbit structure, the opposite conditions will apply:

Theorem 4.2. The following conditions are equivalent:

(1) $D$ has countably many (or finitely many) infinite walks.

(2) The number $w(m)$ of walks in $D$ of length $m$ has polynomial growth with $m$.

(3) $A$ has dominant eigenvalue $\lambda \leq 1$.

(4) No vertex of $D$ is on more than one cycle.

The proofs of the theorems will require several lemmas.

Lemma 4.3. The dominant eigenvalue $\lambda$ of $A$ is $>1$ if and only if $w(m)$ has exponential growth with $m$. The dominant eigenvalue $\lambda$ is $\leq 1$ if and only if $w(m)$ has polynomial growth with $m$.

Proof. If $\lambda>1$, let $v$ denote an eigenvector corresponding to $\lambda$ with all entries nonnegative, and let $u$ denote the vector with all entries 1 . Then $w(m)=$ $\left\langle u, A^{m} u\right\rangle$ using the standard inner product. But for some constant $d>0$, 
$u \geq d v$ (i.e., $u_{i} \geq d v_{i}$ for all $i$ ). Hence $w(m) \geq\left\langle u, A^{m} d v\right\rangle=d\left\langle u, \lambda^{m} v\right\rangle=$ $d\langle u, v\rangle \lambda^{m}$, and so $w(m)$ grows exponentially with $m$.

If $\lambda \leq 1$, put $A$ into Jordan normal form. It is then clear that $w(m)=$ $\left\langle u, A^{m} u\right\rangle$ is a finite sum of terms of form $C m^{r} \mu^{m}$ where $\mu$ is an eigenvalue of $A$ and $C$ and $r$ are constants. Since $|\mu| \leq \lambda \leq 1$, it follows that such a term is bounded by $|C| m^{r}$. Hence $w(m)$ has polynomial growth with $m$.

Since in any case $\lambda \geq 0$, the above cases are exhaustive and the lemma follows.

Lemma 4.4. The binomial coefficient $C(2 r, r)$ has exponential growth with $r$.

Proof. By Stirling's formula [AS, p. 257], for $x>0$ there exists $\theta$ satisfying $0<\theta<1$ such that $x !=(2 \pi)^{1 / 2} x^{x+1 / 2} \exp (-x+\theta /(12 x))$. Since $C(2 r, r)=(2 r) ! /(r !)^{2}$, we compute that $C(2 r, r) \geq(\pi r)^{-1 / 2} 2^{(2 r)} \exp (-1 /(6 r))$ and the lemma follows easily.

Lemma 4.5. Suppose that the vertex $i$ of $D$ is on two distinct cycles. Then $D$ has uncountably many infinite walks and $w(m)$ has exponential growth with $m$.

Proof. Let $i$ be on the distinct cycles $\sigma=u_{0} u_{1} \cdots u_{a}$ and $\tau=w_{0} w_{1} \cdots w_{b}$ where $u_{0}=u_{a}=w_{0}=w_{b}=i$, and $a$ and $b$ are the lengths of the respective cycles. Given an infinite sequence $v=v_{1} v_{2} \cdots$ where each $v_{i}$ is either 0 or 1 , we construct an infinite walk $V_{1} V_{2} \cdots$ by concatenation of the strings $V_{i}$ of symbols where $V_{i}=u_{0} u_{1} \cdots u_{a-1}$ if $v_{i}=0$ and $V_{i}=w_{0} w_{1} \cdots w_{b-1}$ if $v_{i}=1$. It is clear that the walks so constructed are all distinct; and since the set of such $v$ is uncountable, we have thus exhibited uncountably many infinite walks in $D$.

Moreover, if $m=r a+q b$ where $r$ and $q$ are positive integers then any sequence $v_{1} v_{2} \cdots v_{r+q}$ of zeroes and ones yields as above a walk in $D$ of length $m$. The number of such walks is the binomial coefficient $C(r+q, r)$. If we choose $q=r$, then we obtain $w(r a+r b) \geq C(2 r, r)$. But $C(2 r, r)$ grows exponentially with $r$ by (4.4); so there exist constants $D$ and $\lambda$ such that $w(r a+r b) \geq D \lambda^{r}$. Now suppose that $m$ is an arbitrary positive integer and choose $r=[m /(a+b)]$, (the greatest integer). Since $w(m)$ is the number of walks of length $m$ and $m \geq r a+r b$, it is clear that $w(m) \geq w(r a+r b)$. But since $r \geq(m /(a+b))-1$, it follows that $w(m) \geq D \lambda^{r} \geq \bar{D} \lambda^{(m /(a+b))-1}=$ $(D / \lambda)\left(\lambda^{(1 /(a+b))}\right)^{m}$. Hence $w(m)$ has exponential growth with $m$.

Lemma 4.6. Suppose that no vertex of $D$ is on two distinct cycles. Then $D$ has only countably many (or finitely many) infinite walks and $w(m)$ has polynomial growth with $m$.

Proof. Let $\lambda=v_{0} v_{1} \cdots v_{m}$ be a walk of length $m$ in $D$. If two vertices among $v_{0}, \ldots, v_{m}$ are the same, clearly $\lambda$ merely follows the unique cycle containing those vertices between the occurrences of the two vertices. Suppose that the cycles of $D$ are indexed by $i$, that the $i$ th cycle has length $k_{i}$, and that $\lambda$ has exactly $n_{i}$ copies of the $i$ th cycle. It is clear that the walk $\lambda$ is determined by $v_{0}, v_{m}$, the numbers $n_{i}$, and the endpoints of each arc in $\lambda$ which goes between different strong components of $D$. (The strong components of $D$ are the equivalence classes of the vertices under the equivalence $\sim$ that $v \sim w$ if and only if there is a walk, possibly of length 0 , from $v$ to $w$ and also a 
walk from $w$ to $v$.) Let $a$ be the total number of arcs of $D$. Note that if $p+1$ denotes the number of strong components of $D$, then there are at most $p$ such arcs in $g$. The number of choices for these arcs is then at most $L=\max \{C(a, 0), C(a, 1), \ldots, C(a, p)\}$. Let the number of cycles be $Z$ and the number of vertices of $D$ be $v$. The number of choices for each $n_{i}$ is at most $(m+1)$ since $n_{i}$ could be $0,1, \ldots$, or at most $m$. Then clearly $w(m) \leq v^{2} L(m+1)^{Z}$. Thus $w(m)=O\left(m^{Z}\right)$ and hence has polynomial growth with $m$.

An easier analysis shows that there are at most countably many infinite walks in $D$. Each infinite walk must have precisely one cycle which is repeated infinitely often, say the $i$ th cycle, which occurs starting at the $j$ th position in $g$. There are only finitely many possibilities for $v_{0} v_{1} \cdots v_{j-1}$, and there are only countably many choices for $i$ and $j$. Hence there are only countably many infinite walks in $D$.

The above lemmas provide the heart of the proof of Theorems (4.1) and (4.2), the other arguments being trivial. For example, (2) implies (4) in (4.2) since if a vertex is on more than one cycle, then by $(4.5) w(m)$ must have exponential growth and not polynomial growth with $m$. Similarly it is easy to show (2) implies (4) in (4.1) and (1) implies (4) in both theorems.

\section{SUFFICIENT CONDITIONS FOR RICH ORBIT STRUCTURE}

In this section we give some conditions implying rich orbit structure. Typically we utilize the characterization in (4.1) that there be a vertex on more than one cycle.

Lemma 5.1. Suppose $Q$ is a digraph satisfying that for every vertex $v$ there are at least two distinct vertices $u$ and $w$ such that $u \rightarrow v$ and $w \rightarrow v$. Then some vertex of $Q$ is on more than one cycle.

Proof. If we choose any vertex $v_{0}$ we may find a vertex $v_{1}$ such that $v_{1} \rightarrow v_{0}$. Then we may find a vertex $v_{2}$ such that $v_{2} \rightarrow v_{1}$. Continuing in this manner, we may find an arbitrarily long walk in $Q$. Since $Q$ has only finitely many vertices, a long walk must contain a cycle. Thus $Q$ contains at least one cycle.

Suppose that no vertex of $Q$ is on more than one cycle. Form the condensation digraph $G$ [RF, p. 53] whose vertices are the strong components of $Q$ and which possesses an arc from the component of $v$ (denoted [v]) to the component of $w$ (denoted [w]) if and only if there is an arc in $Q$ from some vertex in $[v]$ to some vertex in $[w]$. Since no vertex of $Q$ is on more than one cycle, each vertex of $G$ corresponds to either a single cycle of $Q$ or a single vertex of $Q$. Moreover, $G$ has the property that for each vertex $w$ of $Q$ there is at least one vertex $[v]$ of $G$ so $[v] \rightarrow[w]$ in $G$, since at most one of the two arcs to $w$ in $Q$ could be in the cycle at $w$. Hence as in the first paragraph we may find an arbitrarily long walk and hence conclude that $G$ has a cycle. Yet it is well known that a condensation digraph is acyclic [RF]. This contradiction proves the lemma.

Proposition 5.2. Suppose $K$ is a connected oriented triangulated manifold of dimension $k$, and $f$ is a skeletal map with degree other than 0,1, and -1 . 
Then the iteration digraph $D^{k}$ has some vertex on more than one cycle, and $f$ exhibits rich orbit structure.

Proof. The map $f$ induces a linear map $f_{*}$ of chain complexes. If $[K] \in$ $C_{k}(K)$ denotes the orientation class of $K$, then $f_{*}([K])=d[K]$, where $d$ is the degree of $f$. But $[K]$ is the sum of the $k$-simplexes of $K$, suitably oriented. Since $f$ is skeletal, for each $k$-simplex $I$ we must have that $f_{*}(I)$ is also a sum of $k$-simplexes, namely those $k$-simplexes $J$ suitably oriented such that $I \rightarrow J$. Hence each $k$-simplex $J$ must have at least $|d| k$-simplexes $I$ such that $I \rightarrow J$. By hypothesis, $|d| \geq 2$. Now (5.1) and (4.1) prove the proposition.

Definition. If $D$ is a digraph with vertex set $V$ and $S$ is a subset of $V$, then the generated subdigraph $Q$ from $S$ is the digraph with vertex set $S$ and with $v \rightarrow w$ in $Q$ if and only if $v, w \in S$ and $v \rightarrow w$ in $D$.

Proposition 5.3. Suppose that $D^{k}$ contains a generated subdigraph $Q$ with more than one vertex whose adjacency matrix $A$ satisfies $A^{r}>0$ for some $r$ (i.e., each entry in $A^{r}$ is positive). Then some vertex of $Q$ lies on more than one cycle and $f$ has rich orbit structure.

Proof. Since $A^{r}>0$, given any vertex $v$ of $Q$ there is a walk of length $r$ from $v$ to $v$. If necessary this walk may easily be shortened by deleting duplicated vertices to yield a cycle starting at $v$. Thus every vertex is on at least one cycle. If no vertex lies on more than one cycle, then every vertex is on exactly one cycle. But since $A^{r}>0$, given any two vertices of $Q$ there is a walk in $Q$ from the first to the second. Hence $Q$ consists of a single cycle, say of length $m$, where $m \geq 2$ since there are at least 2 vertices. Choose a vertex $w$ such that there is a walk of length $r+1$ from $v$ to $w$. Then all walks from $v$ to $w$ must have length congruent to $r+1 \bmod m$. This contradicts that since $A^{r}>0$ there is a walk from $v$ to $w$ of length $r$.

\section{Properties of MAPS With Rich orbit STRUCTURE}

If a skeletal map $f$ has rich orbit structure and mild extra conditions then one can conclude that $f$ has properties related to chaotic behavior. In this section we collect a number of such results.

(A) Orbital sensitivity. One of the key properties of chaotic maps is sensitivity to initial conditions. (See [D] or [Gu].) The following condition is closely related. Here $d$ denotes the metric on $K$.

Definition. Let $\varepsilon>0$ and let $S$ be a subset of $K$. We say that $f$ exhibits $\varepsilon$ orbital sensitivity on $S$ provided there exists $\delta>0$ such that for every positive integer $M$ and for every $x \in S$ there exists $y \in K$ and $j>M$ such that $d\left(f^{i}(x), f^{i}(y)\right)<\varepsilon$ for $i=0,1, \ldots, M$ but $d\left(f^{j}(x), f^{j}(y)\right) \geq \delta$.

Definition. The mesh of $K$ is the maximal diameter of a simplex of $K$-i.e., $\sup \{d(x, y)$ : there exists a simplex $I$ of $K$ with $x \in I$ and $y \in I\}$. Recall that a digraph $D$ is strongly connected if, given any two vertices $v$ and $w$ there is a walk from $v$ to $w$.

Proposition 6.1. Let $Q$ be a strongly connected generated subdigraph of $D^{k}$. Assume that the mesh of $K$ is less than $\varepsilon$. Assume that for every infinite walk 
$\sigma=v_{0} v_{1} \cdots$ in $Q$ there exists a positive integer $r$ and a walk $w_{0} w_{1} \cdots w_{r}$ in $Q$ such that $w_{0}=v_{0}$ and $I\left(v_{r}\right)$ and $I\left(w_{r}\right)$ are disjoint. Let $S=\{x \in K$ : there exists an infinite walk $\sigma$ in $Q$ with $x \in C \sigma\}$. Then $f$ exhibits $\varepsilon$-orbital sensitivity on $S$.

Proof. Let $\delta=\inf \{d(x, y)$ : there exist disjoint simplexes $I$ and $J$ corresponding to vertices of $Q$ such that $x \in I$ and $y \in J\}$. Since $K$ has only finitely many simplexes and each is compact, $\delta>0$. Let $x \in C \sigma$ where $\sigma=v_{0} v_{1} \ldots$ is an infinite walk in $Q$. If we are given a positive integer $M$, we may use the walk $v_{M} v_{M+1} \cdots$ to choose an $r$ and a walk $w_{M} w_{M+1} \cdots w_{M+r}$ with $w_{M}=v_{M}$ such that $I\left(v_{M+r}\right)$ is disjoint from $I\left(w_{M+r}\right)$. Now choose any $y \in C v_{0} v_{1} \cdots v_{M} w_{M+1} \cdots w_{M+r}$. Since for $0 \leq i \leq M$ both $f^{i}(x)$ and $f^{i}(y)$ are in $I\left(v_{i}\right)$ and the mesh is less than $\varepsilon$, it follows $d\left(f^{i}(x), f^{i}(y)\right)<\varepsilon$ for $0 \leq i \leq M$. But since $f^{M+r}(x) \in I\left(v_{M+r}\right)$ while $f^{M+r}(y) \in I\left(w_{M+r}\right)$, it follows $d\left(f^{M+r}(x), f^{M+r}(y)\right) \geq \delta$.

Corollary 6.2. Suppose that the mesh of $K$ is less than $\varepsilon$. Let $Q$ be a generated subdigraph of $D^{k}$ for some $k$ such that the adjacency matrix $A$ for $Q$ satisfies $A^{r}>0$ for some $r$. Assume that for each vertex $v$ of $Q$ there is a vertex $w$ of $Q$ such that $I(v)$ and $I(w)$ are disjoint. Let $S$ be the union of the sets $C \sigma$ where $\sigma$ ranges over all infinite walks in $Q$. Then $f$ exhibits $\varepsilon$-orbital sensitivity on $S$.

Proof. $Q$ is strongly connected since $A^{r}>0$. We show that (6.1) applies to $Q$. Suppose that $\sigma=v_{0} v_{1} \ldots$ is an infinite walk in $Q$. Choose a vertex $w$ of $Q$ such that $I\left(v_{r}\right)$ and $I(w)$ are disjoint. Since $A^{r}>0$, there is a walk in $Q$ from $v_{0}$ to $w$ of length $r$.

Corollary 6.3. Suppose that the mesh of $K$ is less than $\varepsilon$ and every point of $K$ lies in some $k$-simplex of $K$. Assume that for every $k$-simplex $I$ there exists a $k$-simplex $J$ disjoint from $I$. Suppose finally that the adjacency matrix $A$ for $D^{k}$ satisfies $A^{r}>0$ for some $r$. Then $f$ exhibits $\varepsilon$-orbital sensitivity on $K$.

Proof. Apply (6.2) to $Q=D^{k}$; we obtain the result once we show $S=K$. So suppose that $x \in K$. Then $x$ lies in some $k$-simplex $I$. If $f$ mapped $I$ into $K^{k-1}$, then there would be no walks in $D^{k}$ starting at $I$, which would contradict that $A^{r}>0$. Hence $f \mid I$ is a homeomorphism and $f(I)$ must be a union of $k$-simplexes of $K$ by Invariance of Domain. It follows that $f(x)$ lies in a $k$-simplex $J$ such that $I \rightarrow J$ in $D^{k}$. If we iterate the argument, we see that there is an infinite walk $\sigma$ in $D^{k}$ such that $x \in C \sigma$. Hence $S=K$.

(B) Entropy. For information about the entropy $h(f)$ of the map $f$ we refer the reader to $[W]$.

Proposition 6.4. Suppose that in the digraph $D^{k}$ the vertex $v$ is on two distinct cycles $\sigma=v_{0} v_{1} \cdots v_{a}$ and $\tau=w_{0} w_{1} \cdots w_{b}$, where $a \leq b$ and $v=v_{0}=v_{a}=$ $w_{0}=w_{b}$. Suppose furthermore there exists $p<a$ such that $I\left(v_{p}\right)$ is disjoint from $I\left(w_{p}\right)$. Then the map $f$ has positive entropy: $h(f)>0$.

Proof. Following [W, p. 169], let $s_{m}(\varepsilon, K)$ denote the largest cardinality of any $(m, \varepsilon)$ separated subset of $K$ with respect to $f$. (A subset $E$ of $K$ is $(m, \varepsilon)$ separated if whenever $x, y \in E$ and $x \neq y$, then there exists an $i, 0 \leq i<m$, such that $d\left(f^{i}(x), f^{i}(y)\right)>\varepsilon$.) 
Assume $\varepsilon$ is less than the minimal distance between any two disjoint $k$ simplexes.

As in the proof of (4.5), for each finite sequence $t_{1} t_{2} \cdots t_{r+s}$ consisting of $r$ 0 's and $s$ 1's we obtain a walk $\mu$ of length $r a+s b$ by following the walks $u_{1}$ then $u_{2} \cdots$ then $u_{r+s}$ where $u_{j}=v_{0} \cdots v_{a}$ if $t_{j}=0$ and $u_{j}=w_{0} w_{1} \cdots w_{b}$ if $t_{j}=1$. These clearly include $C(r+s, r)$ distinct such walks $\mu$. For each such $\mu$ choose a point $x \in C \mu$ and let $E$ be the set of $x$ so obtained. Then, using the hypothesis on disjointness, we easily see that $E$ is $((a r+b s), \varepsilon)$ separated, whence we conclude that $s_{a r+b s}(\varepsilon, K) \geq C(r+s, r)$. Choosing $s=r$, we see that $s_{(a+b) r}(\varepsilon, K) \geq C(2 r, r)$. By the proof of (4.4), it follows

$$
s_{(a+b) r}(\varepsilon, K) \geq(2 \pi r)^{-1 / 2} 2^{(2 r+1 / 2)} \exp (-1 /(6 r)) .
$$

But if $s(\varepsilon, K)=\lim \sup (1 / m) \log s_{m}(\varepsilon, K)$, then $h(f)=\lim _{\varepsilon \rightarrow 0} s(\varepsilon, K)$ so we can obtain that $h(f) \geq(2 \log 2) /(a+b)>0$. This proves the proposition.

\section{(C) Periodic points of high least period.}

Proposition 6.5. Suppose $D^{k}$ has a vertex $i$ which is on two cycles $v_{0} v_{1} \cdots v_{a}$ and $w_{0} w_{1} \cdots w_{b}$ with $i=v_{0}=v_{a}=w_{0}=w_{b}$ and with relatively prime lengths $a$ and $b$. Suppose that there is no face $\mu$ common to all of $I\left(v_{0}\right), I\left(v_{1}\right), \ldots$, $I\left(v_{a-1}\right), I\left(w_{1}\right), \ldots, I\left(w_{b-1}\right)$ such that $\mu \rightarrow \mu$. If $p$ is a prime greater than $a b$, then $K$ contains a periodic point of least point $p$.

Proof. Since $a$ and $b$ are relatively prime, any integer $p$ can be expressed as $p=r a+s b$ for some integers $r$ and $s$. Moreover, if $p>a b$, then $p$ may be so expressed with both $r>0$ and $s>0$. To see this, start with one representation $p=r a+s b$ (with, say, $r<0$ and $s>0$ ) and modify it to the form $p=(r+n b) a+(s-n a) b$. For the smallest positive $n$ for which $r+n b>0$, we will have $s-n a>0$ since otherwise $0<r+n b \leq b$ and $s-n a \leq 0$ force

$$
p=(r+n b) a+(s-n a) b \leq b a+(s-n a) b \leq a b .
$$

Now given prime $p>a b$, write $p=r a+s b$ with $r>0$ and $s>0$ and form a periodic infinite walk $\sigma$ whose period is obtained by following the cycle of $v$ 's $r$ times and then the cycle of $w$ 's $s$ times. The least period of $\sigma$ is then $r a+s b=p$. Choose a periodic point $x \in C \sigma$ by (3.1). By the assumption on faces $\sigma$ has no restriction of period 1, whence by (3.4) $x$ must have least period $p$.

In general, if a periodic point $x$ has least period $p$, then the standard walk $\phi(x)$ may have least period much smaller then $p$. If $f$ does not have rich orbit structure, it is still possible that $f$ has periodic points of arbitrarily high least period. For one-dimensional complexes, however, this situation will not occur:

Proposition 6.6. Suppose that $K$ is a one-dimensional cellular complex and $f$ is a skeletal map which does not have rich orbit structure. Then there is an upper bound on the least periods of all periodic points; i.e., there exists $M>0$ such that if $x$ is a periodic point then the least period of $x$ is less than $M$.

Proof. There are only finitely many 0 -simplexes, hence only finitely many periodic points in $K^{0}$. If $x$ is a periodic point not in $K^{0}$, then $\phi(x)$ is a periodic 
walk in $D^{1}$. By (4.2) there are only finitely many periodic walks in $D^{1}$. By (3.6) for each such walk there is an upper bound on the least period of any corresponding periodic point. The result follows.

We recall the definition of "chaos" from [D, p. 50]: A map $f: S \rightarrow S$ is chaotic on $S$ if (1) $f$ has sensitive dependence on initial conditions; (2) $f$ is topologically transitive; and (3) the periodic points of $f$ are dense in $S$.

Corollary 6.7. If $f$ is a skeletal map on a one-dimensional complex $K$ and $f$ does not have rich orbit structure, then $K$ contains no invariant subset on which $f$ is chaotic.

Proof. By (6.6) there is an upper bound $M$ to the periods of all periodic points of $f$. Hence all periodic points of $f$ are fixed points of $f^{M !}$. If $f$ were chaotic on the invariant set $S \subseteq K$, then the density of the periodic points would imply that $f^{M !}$ would be the identity map on $S$. Hence all points of $S$ would be periodic. Since $f$ is topologically transitive on $S$, it would then follow that $S$ consists of a single orbit and is therefore finite. But no finite set can show sensitive dependence on initial conditions.

\section{EXTENSIONS}

The notion of skeletal map was chosen in order to obtain fairly complete results. In many situations, however, some weakening may be permitted in the hypotheses. This section concerns one very extreme weakening.

Definition. Suppose that $M$ is a metric space and $K \subset M$ is a cellular complex. Let $f: M \rightarrow M$ be a continuous map. The iteration digraph $D$ of $f$ on $K$ is the digraph with one vertex $v$ for every simplex $I(v)$ of $K$ and with an arc from $v$ to $w$ (written $v \rightarrow w$ ) if and only if $f(I(v)) \supseteq I(w)$. If $\sigma=v_{0} v_{1} \cdots$ is a walk in $D$, then as before $C \sigma=\left\{x: f^{i}(x) \in I\left(v_{i}\right)\right.$ for each $\left.i\right\}$.

Proposition 7.1. Let $D$ be the iteration digraph of $f$ on $K$. If $\sigma=v_{0} v_{1} \cdots$ is $a$ (finite or infinite) walk in $D$, then $C \sigma$ is compact and nonempty.

The proof is identical with that of (2.5). Note however that $D$ may have few or no walks. For walks $\sigma$ of finite length in $D^{k}$ (where $D^{k}$ is the subdigraph of $D$ generated by vertices corresponding to simplexes of dimension $k$ ), the set $C \sigma$ need not be a cell, and many results stated in $\S 3$ will not be true in this context. We do have, however, the following result:

Proposition 7.2. Let $\sigma=v_{0} v_{1} \cdots$ be a periodic walk in $D$ of least period $p$. Suppose that for each $i, f \mid I\left(v_{i}\right)$ is one-to-one (hence a homeomorphism onto its image). Then $C \sigma$ contains a periodic point $x$ of period $p$. If $x \in \operatorname{int}\left(I\left(v_{0}\right)\right)$, then $x$ has least period $p$.

Proof. Under these hypotheses $f^{p}: C v_{0} v_{1} \cdots v_{p} \rightarrow I\left(v_{p}\right)$ is a homeomorphism. Hence $C v_{0} v_{1} \cdots v_{p}$ is a cell and the same argument used in (3.1) applies.

The map $f$ has rich orbit structure exhibited on $K$ if $D$ has uncountably many infinite walks. The graph theoretical results in $\S \S 4,5$, and 6 still apply. In particular, the results concerning $\varepsilon$-orbital sensitivity may still apply, so that 
rich orbit structure will imply some properties resembling chaos. On the other hand, the absence of rich orbit structure on $K$ will imply very little.

\section{AN EXAMPLE}

This example is one dimensional only for convenience. Let $a>1$ be a fixed constant and define $f(x)=a x \exp (-x)$ for $x \in[0, \infty)$ and $f(\infty)=0$. Here the extended positive axis including $\infty$ is topologized so that $K=[0, \infty]$ may be regarded as a compact interval and $f: K \rightarrow K$ is a continuous map. Note that $x=1$ is the only internal critical point.

First we choose $a$ so that $f(1) \neq 1$ but $f^{2}(1)=1$; thus $a \approx 9.54895$. Give $K$ a cellular structure by choosing $0,1, c=f(1)$, and $\infty$ as the 0 -simplexes; and choose as 1 -simplexes the natural intervals $u=[0,1], v=[1, c], w=$ $[c, \infty]$. Then $f$ is a skeletal map. A computation of the iteration digraph $D$ shows that $f$ does not have rich orbit structure; the only cycles are $u u, v v$, $00,1 c 1$. By (3.6) all periodic points have periods 1 or 2 , and by (6.7) there is no chaos. It is easy to analyze the behavior of points in each simplex.

Next we choose a sufficiently large so that $f^{2}(1)<f^{3}(1)<1<f(1)$. Let $K=\left[f^{2}(1), f(1)\right]$ and give $K$ the 0 -simplexes $f^{2}(1), 1, f(1)$, and the 1 -simplexes $a=[1, f(1)], b=\left[f^{2}(1), 1\right]$. Then $f$ is not skeletal but the extension in $\S 7$ applies. The iteration digraph on $K$ includes cycles $a a$ and $a b a$; hence $f$ has rich orbit structure. We can locate periodic points corresponding to periodic walks in $D$ using (7.2) since $f$ is a homeomorphism on each simplex. There is no walk $\sigma$ in $D$ such that $C \sigma$ contains the point 1 since $f^{2}(1) \in b, f^{3}(1) \in \operatorname{int}(b)$, but $(b, b)$ is not an arc of $D$. Hence all periodic points from a periodic walk $\sigma$ in $D$ are in the interiors of their 1-simplexes and have the same least period as $\sigma$.

Orbital sensitivity does not follow immediately; but we may refine the specified cellular complex structure by instead taking as 1-simplexes the sets which in the previous description were $C a a, C a b$, and $C b a$. We then readily obtain $\varepsilon$-orbital sensitivity, where $\varepsilon$ is the maximum diameter of the sets $C a a, C a b$, and $C b a$. Further refinement can yield $\varepsilon$-orbital sensitivity for smaller $\varepsilon$.

\section{REFERENCES}

[AS] M. Abramowitz and I. A. Stegun, Handbook of mathematical functions, Dover, New York, 1972.

[B] Rufus Bowen, Equilibrium states and the ergodic theory of Anosov diffeomorphisms, Lecture Notes in Math., vol. 470, Springer-Verlag, Berlin, 1975.

[CE] P. Collet and J.-P. Eckmann, Iterated maps on the interval as dynamical systems, Birkhäuser, Basel, 1983.

[D] R. L. Devaney, An introduction to chaotic dynamical systems, Benjamin, Menlo Park, 1986.

[Ga] F. R. Gantmacher, The theory of matrices, vol. 2, Chelsea, New York, 1959.

[Gu] J. Guckenheimer, Sensitive dependence on initial conditions for one-dimensional maps, Comm. Math. Phys. 70 (1979), 133-160.

[MT] J. Milnor and W. Thurston, On iterated maps of the interval. I, II, preprint, Princeton Univ., 1977.

[P] W. Parry, Symbolic dynamics and transformations of the unit interval, Trans. Amer. Math. Soc. 122 (1964), 368-378.

[RF] D. F. Robinson and L. R. Foulds, Digraphs: theory and techniques, Gordon and Breach, New York, 1980. 
[S] E. H. Spanier, Algebraic topology, McGraw-Hill, New York, 1966.

[W] P. Walters, An introduction to ergodic theory, Springer-Verlag, New York, 1982.

Department of Mathematics, Iowa State University, Ames, Iowa 50011

E-mail address: s1.sjw@isumvs.bitnet 